\title{
Assessment of the innovative quality of agomelatine through the Innovation Assessment Algorithm
}

Liliana Civalleri ${ }^{1}$, Sabrina Licata ${ }^{1}$

SIFEIT - Italian Association for Research on the Economics and Ethics of Medicines and Therapeutic Procedures, Rome, Italy

\begin{abstract}
Aim: the aim of this study was to assess the innovative quality of a medicine based on agomelatine, authorized by the European Commission through a centralized procedure on 19th February 2009 and distributed in Italy under the brands Valdoxan $^{\circledR}$ and Thymanax ${ }^{\circledR}$.

Methodology: the degree of innovation of agomelatine was determined through the Innovation Assessment Algorithm (IAA), which considers the innovative quality of a medicine as a combination of multiple properties. The algorithm may be represented as a decision tree, with each branch corresponding to a property connected with innovation and having a fixed numerical value. The sum of these values establishes the degree of innovation of the medicine. The IAA is articulated in two phases: the first assesses the efficacy of the drug based on the clinical trials presented in support of the registration application (IAA-efficacy); the second reconsiders the degree of innovation on the basis of the efficacy and safety data resulting from clinical practice once the drug has been placed on the market (IAA-effectiveness).

Results and conclusions: the score obtained for agomelatine was 592.73 in the efficacy phase and 291.3 in the effectiveness phase. The total score for the two phases was 884 , which is equivalent to a good degree of innovation for the molecule.
\end{abstract}

\section{Keywords}

Agomelatine; IAA; Innovation; Efficacy; Effectiveness

\section{INTRODUCTION}

\section{Purpose}

This study was meant to assess the degree of innovation for agomelatine $25 \mathrm{mg}$ coated tablets, a new medicine to be used in major depression therapy. To this end, the Innovation Assessment Algorithm (IAA), developed and presented by Prof. Luciano Caprino and $\mathrm{Dr}$ Pierluigi Russo during the six-month Italian Presidency of the European Union (2003), was utilized. The Algorithm, published in Drug Discovery Today in 2006 [1], considers the innovative quality of a medicine not as a single, distinct property, but as a combination of several properties that may be represented like a decision tree. Each branch of the tree, corresponding to a property connected with innovation, is assigned a fixed numerical value. The sum of these values establishes the degree of innovation of the medicine.

\section{Major depression}

Major depression is a serious disorder which affects every year approximately $5 \%$ of the adult population. It presents features of persistence and interferes, sometimes profound- ly, with an individual's way of thinking, with his behavior, with the sleep-wake cycle, as well as with everyday social and working activities. The severity of the illness and, consequently, the improvement of symptoms after therapy are generally measured through rating scales for depression which have been validated worldwide. Among the most common, are the Hamilton Depression Scale (HAM-D), the Montgomery and Asberg Depression Rating Scale (MADRS), and the Clinical Global Impression Scale (CGI-S). Different kinds of treatments, including medicines, psychotherapy, and electroconvulsive therapy (ECT) are available.

In particular, as far as medicines are concerned, there are several specific classes of proven efficacy, but these need to be taken for long periods of time. Further, they must often be combined in order to have a therapeutic effect on the various symptoms of the disorder and are characterized by side- and/or adverse effects which, although often limited to the first few weeks of treatment, influence patient compliance and require a change of therapy. We may add that the disorder has a high relapse rate.
Corresponding author Liliana Civalleri civalleri@res-servizi.it

\section{Disclosure}

This work was sponsored in unconditional form by Servier Italia S.p.A. and I.F.B. Stroder Srl that in Italy distribute agomelatine with brands Valdoxan ${ }^{\circledR}$ and Thymanax ${ }^{\circledR}$, respectively. 
According to the World Health Organization, major depression is the most frequent cause of disability in the world, afflicting individuals aged 5 and above.

Major depression is characterized by a series of signs and symptoms:

- persistently sad or irritable mood;

- changes in sleep patterns (insomnia, waking up early in the morning);

- changes in appetite and body weight (more often weight loss but also weight gain);

- loss of concentration, memory and attention;

- slow movements or agitation;

- loss of interest or pleasure in activities that are normally pleasurable;

- feelings of guilt, uselessness, hopelessness and emptiness;

- recurring thoughts of death or suicide;

- physical symptoms that do not respond to treatment, such as headaches, digestive problems, persistent pains.

In most cases it is a recurring and episodic disease while it is one of the pathologies with the highest social and individual cost.

\section{The medicine}

Valdoxan ${ }^{\mathbb{R}} /$ Thymanax $^{\circledR}$ is a medicine based on agomelatine, a synthetic analogue of melatonine. Its antidepressive action is performed through the melatonergic MT1 and MT2 receptor agonists and the serotonin 5-HT2C receptor antagonist activity. Valdoxan ${ }^{\circledR} /$ Thymanax $^{\circledR}$ was registered in Europe through a centralized procedure and was authorized (February 2009) for the treatment of major depressive disorders in adults.

The recommended dosage is $25 \mathrm{mg} /$ day. The dosage may be increased to $50 \mathrm{mg}$ /day if there is no improvement of symptoms after 2 weeks. A treatment lasting at least six months is recommended.

The undesired side effects are generally mild or moderate, transitory in nature, and present especially during the first two weeks of treatment. The most common are headaches, nausea, dizziness, drowsiness, increase in hepatic enzymes, and hyperhidrosis.

\section{The algorithm}

The IAA is articulated in two phases: the first assesses the efficacy of the drug based on the clinical trials presented to support the registration application (IAA-efficacy); the second reconsiders the degree of innovation on the basis of the efficacy and safety data deriving from clinical practice once the drug has been placed on the market (IAA-effectiveness).
As far as IAA-efficacy is concerned, access to the algorithm may occur through three different access paths (roots), which represent as many degrees of innovation, in a gradually descending order:

- therapeutic innovation;

- common innovation;

- industrial innovation.

Moving along any one of these paths leads to successive decision nodes, from which multiple alternative choices (tree branches) branch off, each one associated with numerical coefficients progressively decreasing according to the position of the decision node on the branch. For each root, a first, second and third level of development is provided for, and for each level several articulations or branches are provided which relate to:

- the drug's action mechanism;

- the social impact of the disorder;

- the type of research design employed;

- the types of objectives achievable for the patient in terms of recovery, management of the disorder, improvement of symptoms, improved tolerance of the medicine, improved risk-benefit ratio;

- typology of criteria for the assessment of clinical results obtained: hard endpoints and surrogate endpoints, quality of life.

Proceeding within the algorithm from left to right the assessment can be completed and a numerical value can be obtained which represents the sum of numerical coefficients attributed to each decision branch selected, an expression of the medicine's degree of innovation in the pre-registration phase.

After this phase is completed, we can then assess the medicine after its placement on the market (IAA-effectiveness). The properties evaluated during this phase are:

- the types and purposes of post-registration trials;

- in a chronic disease, the length of time of the trials; in an acute/sub-acute disease, the number of patients enrolled;

- the types of criteria for assessing clinical results obtained (hard endpoints and surrogate endpoints, quality of life);

- the types of objectives achievable for the patient in terms of recovery, management of the disorder, improvement of symptoms, improved tolerance of the drug, improved risk-benefit ratio;

- the selection criteria for patients enrolled;

- the size of the clinical trials (Multicenter, National or International);

- the severity and frequency of harmful side effects registered by the pharmacovigilance system and in the context of effectiveness studies. 
In this case too, a score will be obtained that must be added to that obtained during the first phase.

In conclusion, the innovation assessment of a medicine through the IAA allows us to:

- bind the innovation value to the determination of the clinical efficacy (on the pre-registration level) and of the clinical effectiveness (almost always in the postmarketing phase);

- express, through a numerical value, the innovation contribution made by a drug. This value can be established on the basis of a decision algorithm capable of taking into consideration the various elements that contribute to innovation;

- utilize it, in full or in part, both for the purpose of Authorization of Placement on the Market for a medicine, and for re-assessment of the medicine's extent of innovation during the post-marketing period.

\section{Innovation assessment by means of the IAA}

Measurement of the degree of innovation is performed by using a specific software, downloadable from the site of the SIFEIT - Italian Association for Research on the Economics and Ethics of Medicines and Therapeutic Procedures, which enables us to have guided access to assessment of the drug.

\section{METHODS}

\section{IAA-efficacy}

\section{First level}

Access to the algorithm occurs through selection of the general property expressed by the three initial degrees of innovation (roots). In Figure 1, the diagram relating to the first level of the decision tree is shown.

For agomelatine, access to the algorithm occurs through branch B of therapeutic innovation: in fact, agomelatine is a new chemical entity which represents the prototype of a new class, active against a pathology (major depression) for which pharmacological therapies already exist. In fact, those already present on the market are:

- tricyclic antidepressants (TCA);

- monoaminooxidase inhibitors (IMAO);

- selective serotonin reuptake inhibitors (SSRI);

- selective noradrenaline reuptake inhibitors (SNRI);

- dopamine reuptake blocking drugs.

\section{Second level}

\section{Level 2.1 - mechanism of action}

Accessing the IAA through branch B, the first aspect to assess is the mechanism of action for which two alternatives are possible (Figure 2). In the case of agomelatine the mechanism of action is new, since it is a melatoner-

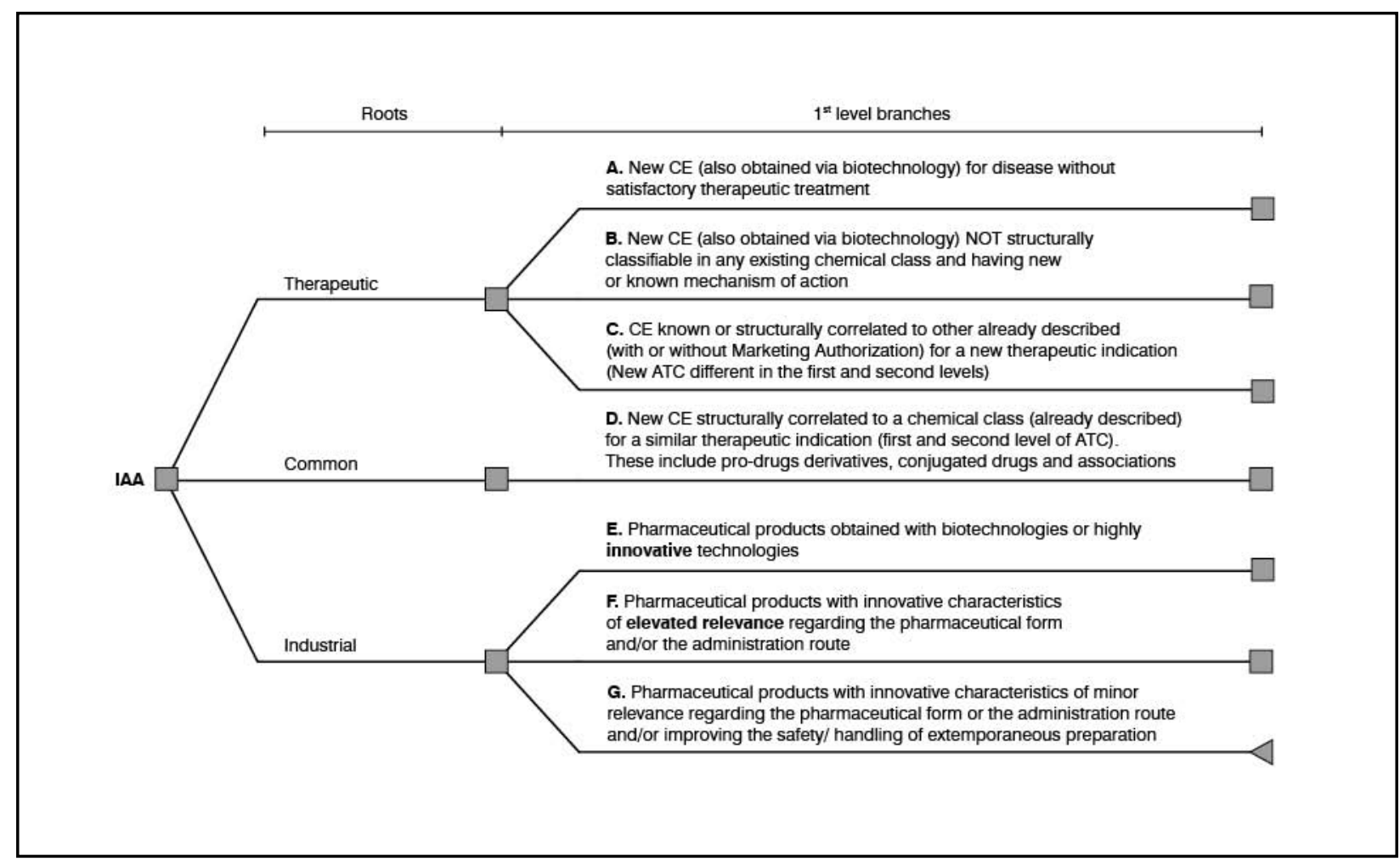

Figure 1. IAA-efficacy: first level of the decision tree ATC $=$ Anatomic Therapeutic Chemical; $C E=$ chemical entity 


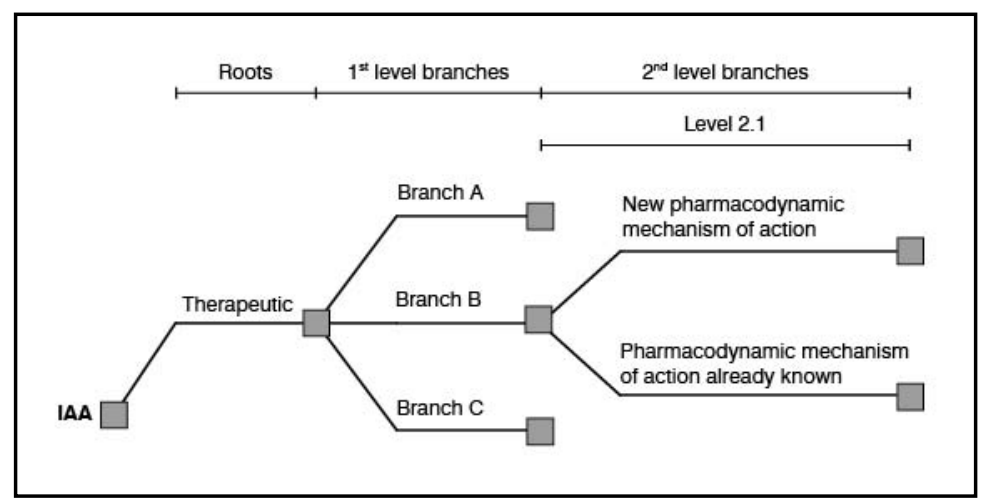

Figure 2. IAA-efficacy, level 2.1: action mechanism

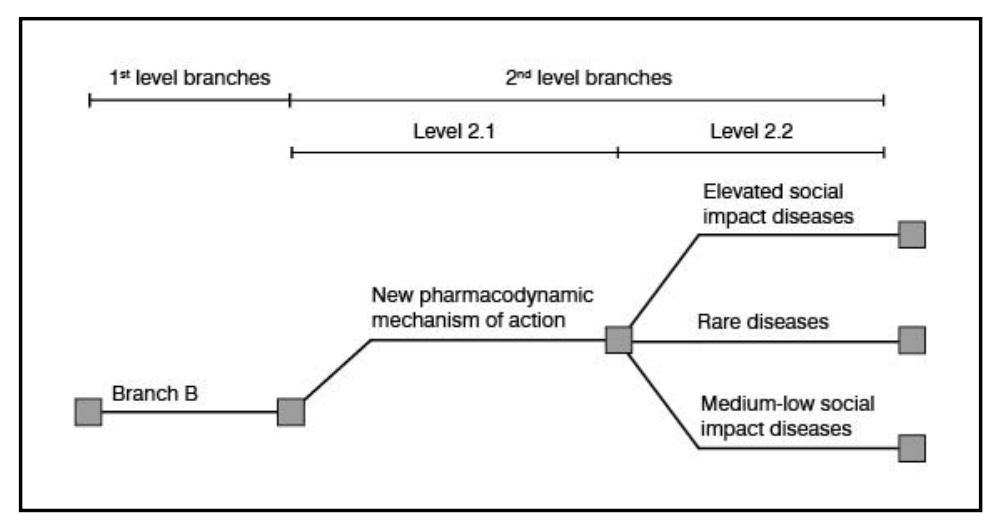

Figure 3. IAA-efficacy, level 2.2: typology of the disorder

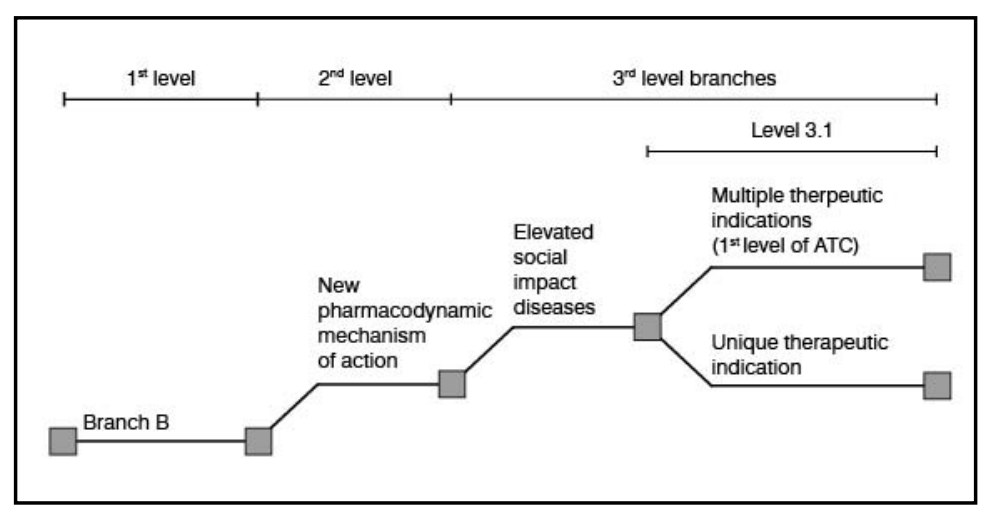

Figure 4. IAA-efficacy, leve/ 3.1: therapeutic indications ATC $=$ Anatomic Therapeutic Chemical

gic agonist (acts on MT1 and MT2 receptors) and, at the same time, is a serotonin 5-HT2C receptor antagonist.

Its antidepressant action depends on its activity on both types of receptors and represents a new concept for the treatment of depression [2-4]. In addition, the molecule has shown anxiolytic effects as well as effects of resynchronizing circadian rhythms, due to the melatoninergic activity.

\section{Level 2.2 - typology of the disorder}

The second node of the first level of the decision tree (Figure 3) refers to the social impact of the disease for which the drug is indicated. Due to the high degree of disability and its high social and individual cost, major depression falls within the definition of an elevated social impact disease.

\section{Third level}

Level 3.1 - therapeutic indications

Proceeding along the decision tree to the third level, the first assessment to be made (Figure 4) relates to the possibility of multiple therapeutic indications within the first level of ATC. Agomelatine has only one indication: the treatment of major depression episodes in adults.

\section{Level 3.2 - type of clinical study designed employed}

The level 3.2 algorithm provides for selection of the branch with regard to the types of trials and requires a guideline opinion (excellent, sufficient, poor) on the performance and/or data contained within them (Figure 5).

The main clinical trial documentation on agomelatine involves over 4,000 subjects diagnosed with major depression who took agomelatine. It involves randomized, double blind trials, mostly multicenter, performed vs. a placebo or vs. an active drug.

Trials performed vs. placebo include a study for verifying the minimum effective dose (dose-finding study) [5,6]: a short term (8 weeks), randomized, double blind study performed on 711 patients. Three different dosages of agomelatine were compared $(1,5$, and $25 \mathrm{mg}$ ) using paroxetine as an internal control with the variation of the score on the HAM-D scale used as the main efficacy measurement. The lowest doses proved to have an effect similar to the placebo. The $25 \mathrm{mg}$ dose was statistically more effective than the placebo, both compared to the HAM-D score and compared to other assessment criteria (Montgomery-Asberg Depression Rating Scale, Clinical Global Impression-Severity of Illness). There were no differences between agomelatine and paroxetine. Two studies with agomelatine $25 \mathrm{mg}$ or $50 \mathrm{mg}$ vs. placebo [6-8], both short term (six week) multicenter, randomized, double blind, are included: the first [7] performed on 211 subjects, the second [8] on 235 subjects. For subjects who showed no improvement with agomelatine $25 \mathrm{mg}$ at two weeks, the dose was increased to $50 \mathrm{mg}$ through an electronic system that allowed the double blind status to be maintained. In this case too, the main assessment was based on the variation of the score on the HAM-D scale. In both studies, agomelatine was significantly more effective than the placebo. 
In the CHMP Assessment Report presented to the EMA [9], three more short term studies were described, all with active internal control (paroxetine or fluoxetine) in which there was no statistically significant difference between agomelatine and the placebo. In this regard, the discussion reports that agomelatine probably has a lower efficacy than other antidepressants and that further studies, on a larger scale, are necessary to confirm the efficacy of the medicine. Lastly, a specific study was performed to assess maintenance of the pharmacological effect and the time elapsed until reappearance of the disorder [10]: a long term study (for 24 weeks after a period of 8-10 weeks of openly taking the drug), multicenter, randomized, double blind, on 339 responders in the first phase; the assessment was based on the time elapsed until reappearance of the disorder. The results show a statistically significant difference in the time until relapse, in favor of agomelatine.

The symptoms evaluated through the HAM-D scale reappeared in $21 \%$ of the patients treated with agomelatine over the 24 weeks (34 out of $165)$, compared with $41 \%$ of the patients treated with the placebo (72 out of 174). No differences were reported between subjects with serious and/or moderate/mild depression. A long term study, preceding the one described above, did not show statistically significant differences between agomelatine and the placebo.

Among the studies performed vs. active medicines, we report two studies vs. venlafaxine $[11,12]$ : short term (6 weeks for the first, 12 weeks for the second), multicenter, randomized, double blind studies. The first [11] involved 332 subjects (165 treated with agomelatine $25-50 \mathrm{mg}, 167$ with venlafaxine $75-150 \mathrm{mg}$ ). The purpose of the study was to assess the improvement of sleep patterns (understood as falling asleep, quality of sleep, number of times patient wakes up at night), measured through an appropriate scale, the Leeds Sleep Evaluation Questionnaire (LSEQ) and the total antidepressant effect, measured by the HAM-D and the CGI$\mathrm{S}$. Because agomelatine is a drug that also acts on the sleep-wake cycle, the beneficial effect is without a doubt better for this drug than for the comparison drug. The improvement of the quality of sleep was also important for the antidepressant effect. The second study [12], involved 277 subjects (137 treated with agomelatine $50 \mathrm{mg}, 140$ with venlafaxine $75-50 \mathrm{mg}$ ). The purpose of the study was to assess the harmful side effects typical for most antidepressant drugs, the deterioration of sexual functioning (through the Sex FX scale), and the antidepressant efficacy (through the MADRS, CGI scale). Agomela-

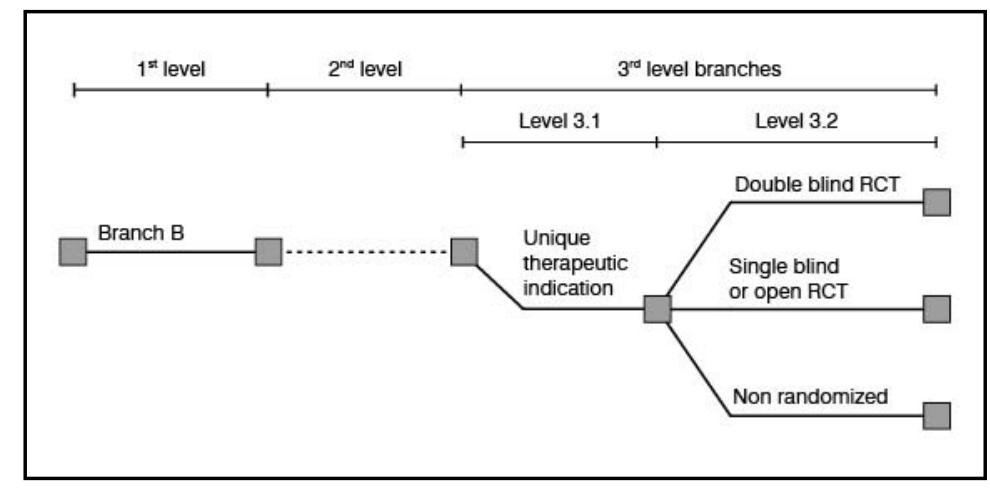

Figure 5. IAA-efficacy, level 3.2: types of clinical studies $\mathrm{RCT}=$ Randomized Clinical Trial

tine use results in a statistically lower occurrence of sexual disorders compared to venlafaxine. There is no significant difference in the decreased MADRS score. A study vs. sertraline [13] was also performed: a short term (6 weeks), multicenter, randomized, double blind study on 313 subjects, (154 agomelatine $25-50 \mathrm{mg}$ vs. 159 sertraline $50-100 \mathrm{mg}$ ); the principal assessment was based on the sleep-wake cycle and on the HAM-D scale. The results show a statistically significant antidepressant effect for agomelatine, calculated on different scores of the HAM-D scale, starting from the second week of treatment.

As stated above, the branch of the decision tree to be selected for level 3.2 has to do with double blind studies. The results obtained are deemed sufficient, although it may be necessary to perform in depth clinical trials in order to verify the efficacy of the drug, for use with elderly patients and for any possible interactions or effects that can only be verified in clinical practice.

\section{Level 3.3 - population}

involved in the studies

This level assigns a score relative to the age groups of the population involved in the studies performed (Figure 6): as stated above, subjects involved in studies with agomelatine are between the ages of 18 and 60 years of age (adults). In the CHMP Assessment Report presented to the EMA [9], a trial performed on subjects $>65$ years of age is described which did not show a significant difference between agomelatine and the placebo. The desirability of performing a post-registration study for these types of subjects is expressed. The branch to follow on the decision tree is that relating only to the adult population.

\section{Level 3.4 - types of achievable objectives}

This level allows selection between treatment or management of the disease (in the case of chronic disease) and the improvement of symptoms (Figure 7). From what has been 
reported, agomelatine appears to have significantly greater therapeutic efficacy compared with the placebo or with the comparison drug in the treatment of Major Depression, in addition to controlling some of its specific symptoms. At this level of the algorithm, the selected branch is therefore that having to do with treatment of the disease.

This level assigns a score based on the types of outcomes (Figure 8) and in this instance too a guideline opinion is required (excellent, sufficient, poor).

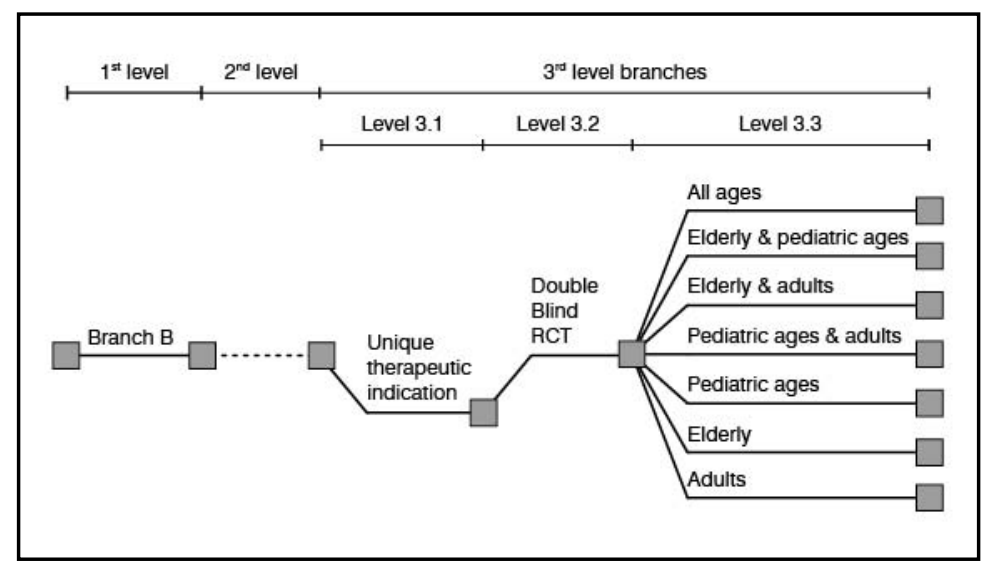

Figure 6. IAA-efficacy, level 3.3: types of populations involved in the clinical trials

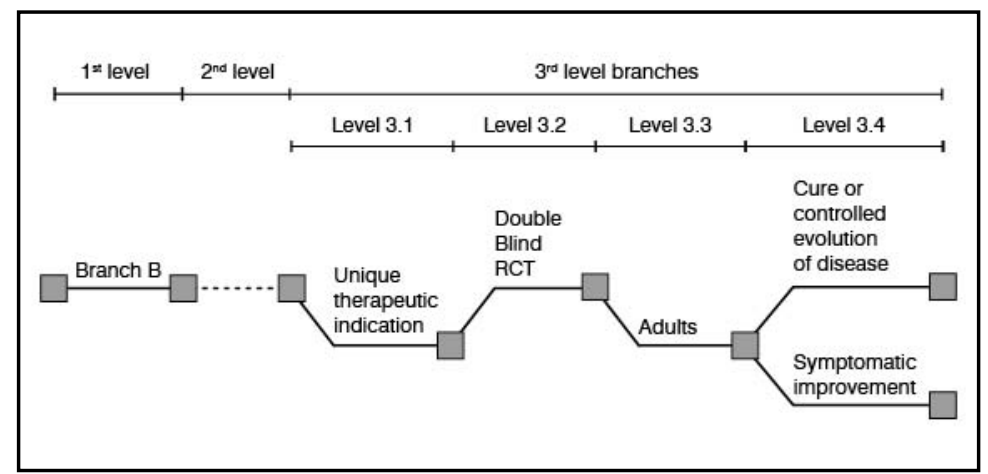

Figure 7. IAA-Efficacy, level 3.4: typology of achievable objectives $\mathrm{RCT}=$ Randomized Clinical Trial

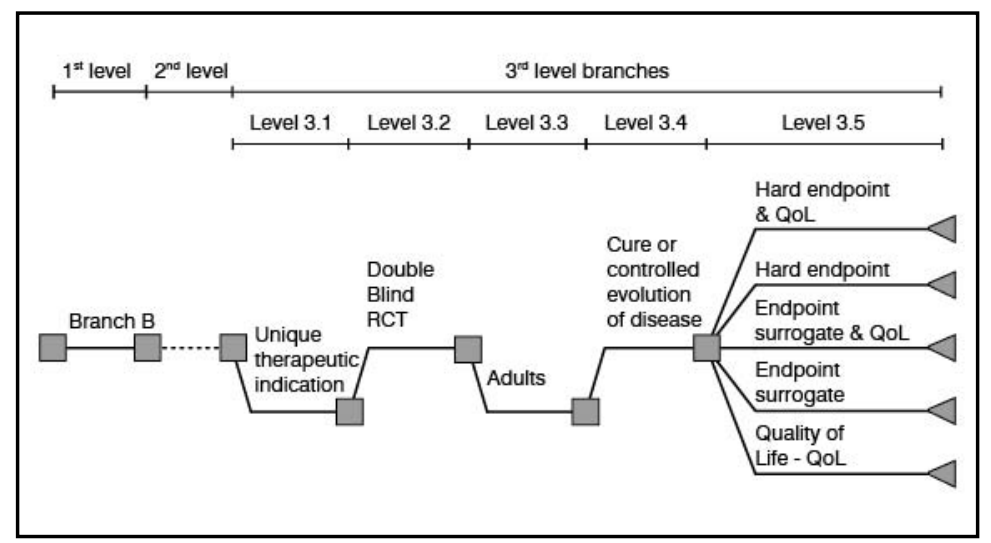

Figure 8. IAA-Efficacy - level 3.5: type of clinical outcome obtained $\mathrm{RCT}=$ Randomized Clinical Trial
Level 3.5 - type of clinical outcome obtained

From what is reported above, we can see that agomelatine appears to be effective in major depression, both in terms of treatment of the disease and in preventing its recurrence, as well as in terms of improvement of quality of life (normalization of the sleep-wake cycle, improvement of sexual activity). Therefore, the branch to select is that relating to hard end-points and quality of life (HE + QoL). The results were deemed sufficient.

\section{IAA-effectiveness}

The structure of the algorithm in the second phase is similar to that of IAA-efficacy, with several levels corresponding to as many characteristics that contribute to the innovation value of the drug, based on post-marketing clinical trials. IAA-effectiveness starts from a single branch defined by the score obtained at the end of the assessment of the IAA-efficacy route, which develops on three levels. In this case too, a score will be obtained that must be added to the one obtained in the first phase.

\section{First level}

This level has to do with the presence or absence of clinical trials published or performed after authorization for placement on the market was obtained. Five new trials are presented, of which one is versus placebo, three versus a comparison drug, and one versus both a placebo and a comparison drug. The study performed vs. placebo was a short term (8 weeks), multicenter, randomized, double blind trial, performed on 503 patients, affected by episodes of major depression moderate/severe in degree (168 treated with agomelatine $25 \mathrm{mg} /$ day, 169 with agomelatine $50 \mathrm{mg} /$ day and 166 treated with placebo) [14]. The primary endpoint of the study was reduction of the score on the Hamilton Depression Rating Scale $\left(\mathrm{HAM}-\mathrm{D}_{17}\right)$ at the $8^{\text {th }}$ week. The $25 \mathrm{mg}$ dose proved to be statistically more effective than the placebo $(\mathrm{p}=0.01)$; the $50 \mathrm{mg}$ dose also proved to be statistically more effective than the placebo from the $2^{\text {nd }}$ to the $6^{\text {th }}$ week of treatment, but not at the $8^{\text {th }}$ week. As to the secondary endpoints, patients treated with agomelatine 25 $\mathrm{mg}$ showed a greater percentage of clinical response $(p=0.013)$ and clinical remission $\left(\right.$ HAM-D $\left.{ }_{17}\right)$, than the group treated with the placebo; the same can be said for clinical improvement (CGI-I). The $50 \mathrm{mg}$ dose did not demonstrate any statistical significance. The Hospital Anxiety and Depression Scale (HADS) score and the Leeds Sleep Evaluation Questionnaire (LSEQ) quality of sleep score, improved significantly, compared to 
the placebo, both with the $25 \mathrm{mg}$ dose and with the $50 \mathrm{mg}$ dose. Both agomelatine doses were well tolerated, with percentages of adverse events that are identical for the three treatment groups. With the $50 \mathrm{mg}$ dose, a temporary, but clinically relevant, increase of hepatic aminotransferase was found in 5 patients. The studies performed vs. the active drug, comprise a short term (8 week), multicenter, international, randomized, double blind study performed on 515 pts (252 treated with agomelatine $25-50 \mathrm{mg} /$ day and 263 with fluoxetine $20-40 \mathrm{mg} /$ day), with severe degree MDD (HAM-D ${ }_{17}$ basal values $\geq 25$ and CGI $\geq 4$ ) [15]. The main objective of the trial was to reduce the HAM-D ${ }_{17}$ scale score after 8 weeks of treatment. The secondary efficacy assessments were based on CGI-S and HAM-A; an additional secondary assessment that concerned the quality of sleep was based on LSEQ. The average decrease of the total HAM-D ${ }_{17}$ scale score proved to be statistically greater $(p=0.024)$ in the group treated with agomelatine compared to the fluoxetine group. Between the secondary endpoints, the percentage of responders at the $8^{\text {th }}$ week ( HAM-D ${ }_{17}$ and CGI-I) proved to be greater in the agomelatine group; moreover, a statistically significant difference $(p=0.018)$ was found in favor of agomelatine on the HAMD score for sleep items. The two medicines in the trial proved to be well tolerated, with similar percentages of side effects, which occurred independently of the dosage used.

The other short term (6 weeks) study vs. active drug, was a multicenter, international, randomized, double blind study performed on 313 patients (154 treated with agomelatine $25-50 \mathrm{mg}$ /day and 159 with sertraline 50-100 mg/day), with MDD of a moderate/ severe degree [16]. The primary objective of the study was evaluation of the Relative Amplitude (RA) of the individual rest-activity cycles (like the sleep-wake cycle index), measured by actigraphy; the secondary objectives, likewise measured by actigraphy, were sleep efficiency (the ratio between total sleeping time and time spent in bed), sleep latency and efficacy on depression and anxiety symptoms evaluated with the HAM-D ${ }_{17}$, CGI and HAM-A scales. Agomelatine showed a statistically significant difference compared to sertraline, on the RA of the individual restactivity cycles after the first week $(p=0.01)$, on sleep latency $(p<0.001)$, and on sleep efficiency $(p<0.001)$ from the $1^{\text {st }}$ week to the $8^{\text {th }}$ week, as well as on depression symptoms $(\mathrm{p}<0.05)$ and anxiety symptoms $(\mathrm{p}<0.05)$. The two drugs under study proved to be well tolerated, with similar side effect percentages, except for tiredness $(5.9 \%$ agomelatine and $1.3 \%$ sertraline) and hyperhidrosis (5\% sertraline and $0 \%$ agomelatine).

The long term study (24 weeks) was a multicenter, international, randomized, double blind trial performed on 138 patients (71 treated with agomelatine $25-50 \mathrm{mg}$ /day and 67 with escitalopram 10-20 mg/day), with MDD of moderate/severe degree [17]. The polysomnographic results proved that treatment with agomelatine was associated with a significant reduction in sleep latency compared to escitalopram from the $2^{\text {nd }}$ week up to the end of the assessment period. The efficiency of sleep was improved with agomelatine and, as far as sleep architecture was concerned, the REM (Rapid Eye Movement) latency significantly increased in the escitalopram group in all the visits carried out. In addition, agomelatine significantly preserved the number of sleep cycles compared to the marked decrease observed with escitalopram. The assessments performed with the analog scale showed an improvement of conditions upon awakening and decreased daytime drowsiness, in the group treated with agomelatine. The total score on HAM-D ${ }_{17}$, CGI-I and CGI-S decreased in a similar manner in the two groups. The two medicines under study proved to be well tolerated; agomelatine was associated with a significantly lower incidence of adverse events, as well as adverse events considered to be associated with the treatment by the investigator.

Lastly, the study performed vs. active drug and placebo was a short term ( 8 weeks), randomized, double blind trial, performed on 92 healthy volunteers (23 treated with agomelatine $25 \mathrm{mg} /$ day, 23 with agomelatine $50 \mathrm{mg} /$ day, 23 with paroxetine $20 \mathrm{mg} /$ day and 23 with placebo). The healthy volunteers represented an ideal group of subjects to assess for sexual dysfunction, in that they allowed for elimination of influences linked to depression [18]. The primary objective of the study was assessment of sexual dysfunction (SD) using the validated Psychotropic-Related Sexual Dysfunction Salamanca Sex Questionnaire (PRSEXDQ-SALSEX). At the $8^{\text {th }}$ week, the SD proved to be significantly reduced in the agomelatine groups compared to the paroxetine group $(p<0.0001)$. The percentage of volunteers with moderate or severe SD was $4.5 \%$ in the agomelatine group $25 \mathrm{mg}$, $4.8 \%$ in the $50 \mathrm{mg}$ group, $61.9 \%$ in the paroxetine group and $0 \%$ in the placebo group ( $\mathrm{p} \leq 0.0001$ agomelatine vs. paroxetine). The products under study were well tolerated and the assessments of hormonal profiles (prolactin, total cortisol, free and total testosterone), did not show significant clinical variations compared to the placebo. 


\section{Second Level}

Level 2.1 - representative degree of the studies

This level provides a judgment relative to the international character of the studies and it is articulated in:

- clinical trials based on enrollment of patients in an international context;

- clinical trials based on the enrolment of patients at several trial sites within a single country.

The studies, with the exception of one, were performed within an international context. Therefore, the selection falls on the upper branch.

\section{Level 2.2 - quality of the research design}

The level 2.2 of the algorithm provides for selecting the branch relating to the adequacy of the study protocol depending on the epidemiological characteristics of the disease for which the drug is used. A guideline opinion (excellent, adequate, poor) is required of the research design. All the studies were randomized, prospective, double blind. However, the judgment that is issued is that of adequate, because elderly subjects were lacking within the population considered, and no interactive situations were considered. In addition, with the exception of a 24-week study, the term was never longer than 8 weeks.

\section{Level 2.3 - degree of representativeness of the comparison drug}

The parallel with the drugs selected for comparison, relative to the type of disease treated, may be classified as:

- sufficiently representative;

- not particularly representative;

\begin{tabular}{lcl}
\hline \multicolumn{1}{c}{ Level } & Alternative & Score \\
\hline 2.1 & New action mechanism & 200.00 \\
2.2 & Disease with high social impact & 200.00 \\
Total level 2 & & 400.00 \\
\hline
\end{tabular}

Table I. IAA- efficacy: agomelatine score on level 2, branch B

\begin{tabular}{clc}
\hline Level & \multicolumn{1}{c}{ Alternative } & Score \\
\hline 3.1 & Single therapeutic indication & 16.80 \\
3.2 & Random, double blind controlled clinical trials & 46.34 \\
3.3 & Population group: adults / all ages for whom & 25.80 \\
& drug use is indicated & \\
3.4 & Treatment of the disease & 27.70 \\
3.5 & Type of clinical outcome obtained & 76.09 \\
Total level 3 & & 192.73 \\
\hline
\end{tabular}

Table II. IAA- efficacy: agomelatine score on level 3, branch B
- not representative.

In this case the judgment is sufficiently representative in that the studies were performed versus sertraline, escitalopram, paroxetine and fluoxetine which represent a great portion of the current therapeutic stock in trade for major depression.

Level 2.4 - inclusion/exclusion criteria

The clinical experimentation proposed for IAA-effectiveness assessment may show:

- inclusion and exclusion criteria present in the information given to the patient and the physician;

- other inclusion and exclusion criteria not existing in the information given to the patient and to the physician, that may differentiate the clinical and demographic characteristics of the population evaluated from those regularly exposed to the treatment in clinical practice.

In this case, the inclusion/exclusion criteria described in the information about the drug were respected; therefore the upper branch is selected.

\section{Third level}

The third level is dedicated to chronic disease, therefore we can proceed to the fourth level.

\section{Fourth level}

Level 4.1 - number of patients enrolled

In the presence of an acute or sub-acute disease, the number of patients enrolled is taken into consideration in descending order:

- more than 200 patients;

- between 100 and 200 patients;

- less than 100 patients.

The total number of patients treated with agomelatine in the 5 studies was 580 patients.

\section{Level 4.2 - types of achievable objectives}

This level allows for the selection of either treatment or control of the disease (in case of chronic disease) and the improvement of symptoms. As reported, agomelatine appears to have significantly greater therapeutic efficacy compared to the placebo or to the comparison drug not only in treating major depression by also in controlling some of its specific symptoms. At this level of the algorithm, the upper branch is selected.

\section{Level 4.3 - type of clinical outcome obtained}

This level assigns a basic score to the types of outcomes and in this case as well a guideline opinion is required (excellent, sufficient, poor). As reported above, it can be seen that agomelatine appears effective for major depression, both in terms of treating the disease, and in terms of improvement of sleep 


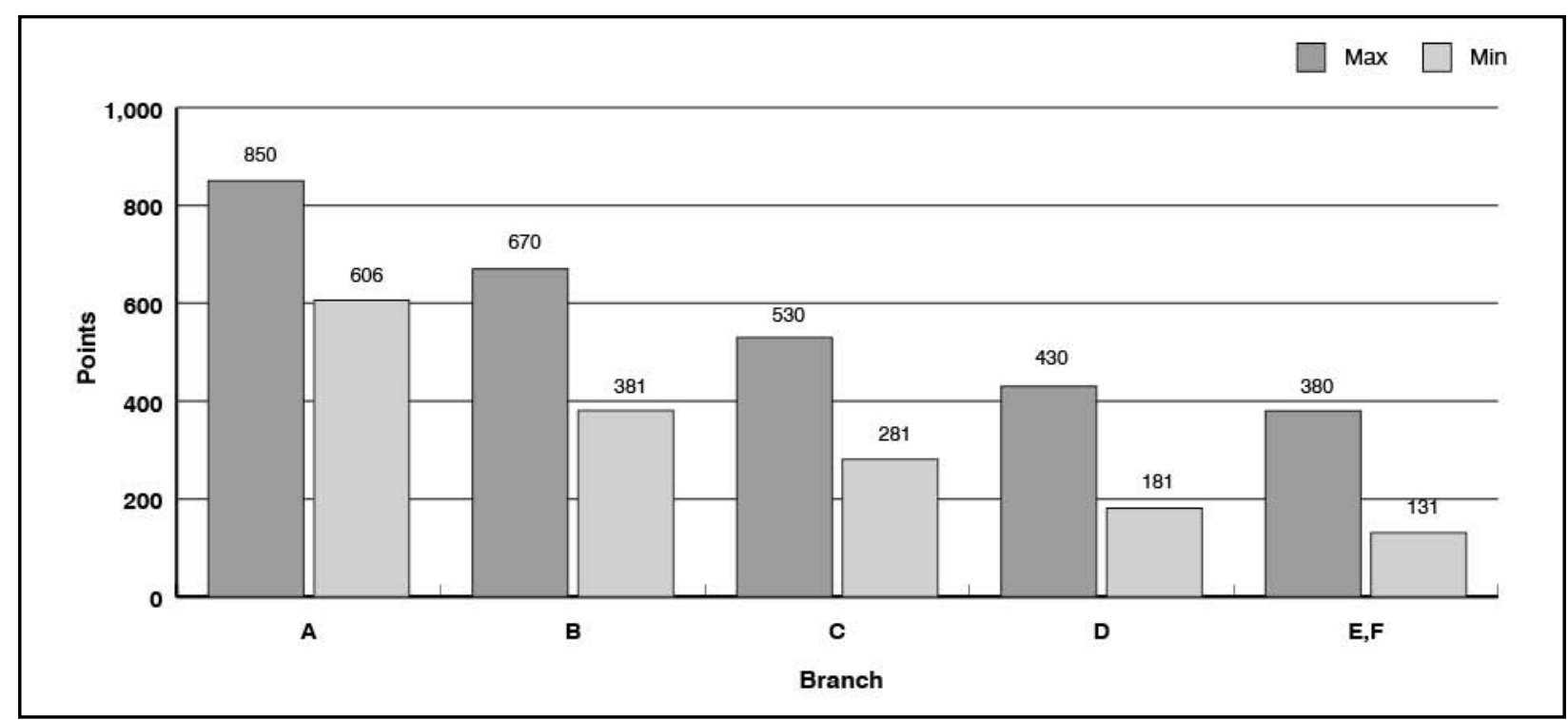

Figure 9. Score obtained for agomelatine for each branch in the efficacy phase

architecture, sexual activity, and quality of life. Therefore, the branch to be selected is that relating to hard endpoints and quality of life $(\mathrm{HE}+\mathrm{QoL})$. The results are deemed to be excellent.

\section{Harmful side effects}

Once the effectiveness assessment has been completed, the algorithm takes into consideration any serious harmful side effects that occurred during the trial. In the case of agomelatine, no serious harmful side effects were reported. Therefore, as far as this assessment is concerned, the score is 0 .

\section{RESULTS}

\section{IAA-efficacy}

In Table I the scores obtained with agomelatine are reported, based on selections made at the second level, proceeding on the decision tree through access to branch B.

On Table II the scores obtained with agomelatine are reported, based on the selections made on the third level.

\section{Assigning the total score}

The scores obtained in the first and second parts were added in order to calculate the final score obtained in the phase relating to efficacy assessment. The final score obtained for agomelatine is 592.73. Figure 9 shows the distribution of the maximum and minimum final scores that were obtained for each branch (A-F). The score decreases as the degree of innovation represented by each branch $(\mathrm{A} \rightarrow$ F) decreases. For agomelatine, access to the algorithm occurs through branch B of therapeutic innovation: in fact, agomelatine is a new chemical entity which represents the pro- totype of a new class, active against a pathology (major depression) for which pharmacological therapies already exist.

Figure 10 shows the score obtained for the drug relative to the maximum and minimum score obtainable through access to branch B.

\section{IAA-effectiveness}

Table III shows the scores obtained for agomelatine based on the selections made at the second level.

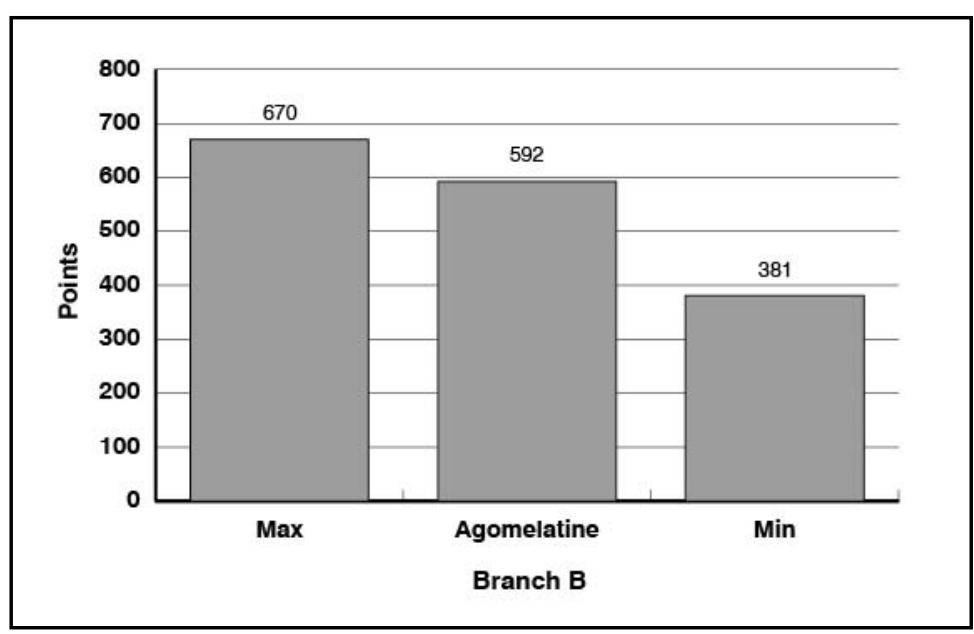

Figure 10. Final score obtained for agomelatine in the efficacy phase

\begin{tabular}{clc}
\hline Level 2 & \multicolumn{1}{c}{ Alternative } & Score \\
\hline 2.1 & International studies & 22.50 \\
2.2 & Quality of the research design & 43.80 \\
2.3 & Representativeness of the comparison drug & 52.50 \\
2.4 & Inclusion/exclusion criteria & 22.50 \\
Total level 2 & & 141.30 \\
\hline
\end{tabular}

Table III. IAA-effectiveness: agomelatine score on level 3 


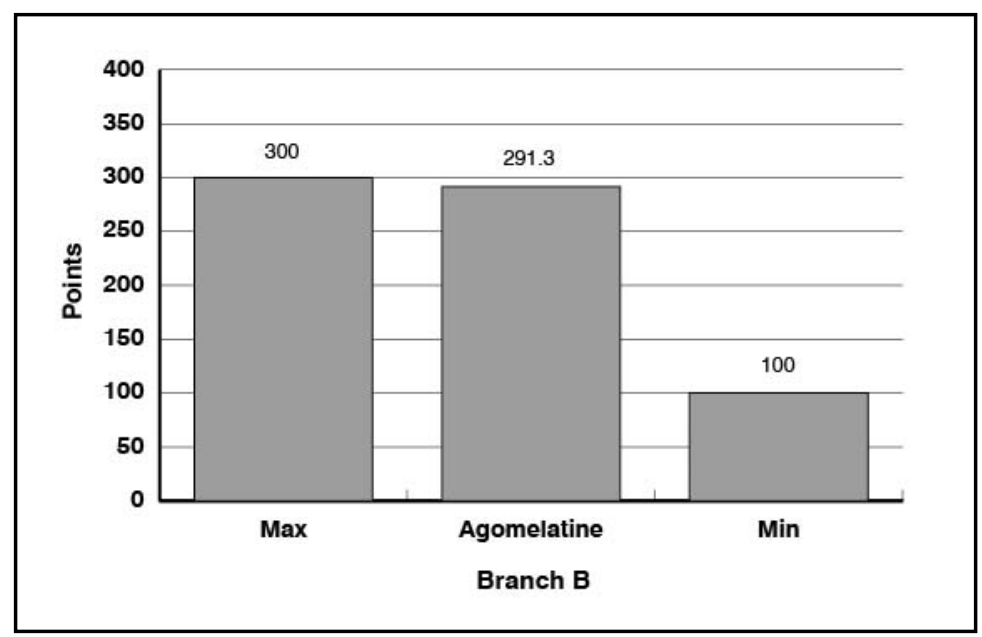

Figure 11. Score obtained for agomelatine in the effectiveness phase

\begin{tabular}{clc}
\hline Level 4 & \multicolumn{1}{c}{ Alternative } & Score \\
\hline 4.1 & Number of patients enrolled & 67.50 \\
4.2 & Therapeutic results & 22.50 \\
4.3 & Clinical results observed & 60.00 \\
Total level 4 & & 150.00 \\
\hline
\end{tabular}

Table IV. IAA-effectiveness: agomelatine score on level 4

Table IV shows scores obtained for agomelatine based on selections made at the fourth level.

\section{Assigning the total score}

The scores obtained on the second and fourth levels were added to calculate the final score obtainable in the phase relating to the effectiveness assessment. The final score obtained for agomelatine is 291.3. Figure 11 shows the agomelatine score in the effectiveness phase relative to the maximum and minimum scores obtainable in the post-marketing assessment (not including ADV).

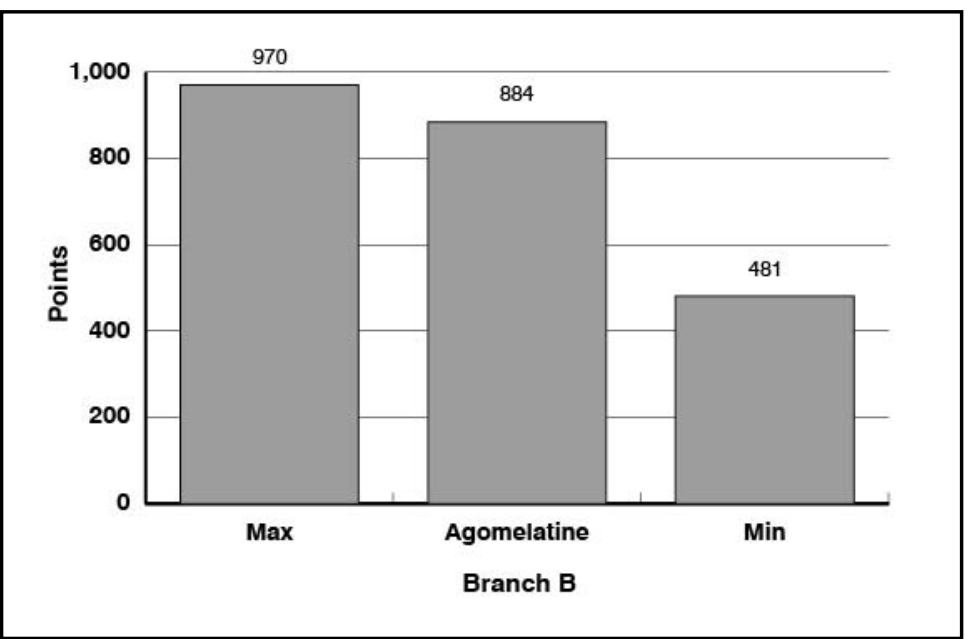

Figure 12. Score obtained for agomelatine in the efficacy phase + the effectiveness phase

\section{Assigning the total score (IAA- efficacy + IAA-effectiveness)}

The total score obtained for IAA-effectiveness is added to the score obtained initially in the IAA-efficacy phase. Agomelatine received a total score of 884 . Figure 12 shows the score obtained for agomelatine in the efficacy phase + the effectiveness phase relative to maximum and minimum obtainable scores through access to branch B of the first phase.

\section{DISCUSSION}

The innovation assessment algorithm assigns the highest score to new entities capable of treating pathologies for which no alternative therapies exist at the time (branch A of the Therapeutic Innovation). The maximum score that may be obtained by the efficacy assessment is 850 points. Access to the algorithm through branches $\mathrm{E}$ and $\mathrm{F}$ of Industrial Innovation leads to obtaining lower scores (from a maximum of 380 points to a minimum of 131). Agomelatine obtained a score of 592.73 which expresses a good degree of innovation. The factor that made the greatest impact on the score was the new chemical structure of the molecule, not related to any pharmacological class currently used for major depression, with a new action mechanism (melatoninergic agonist). This element, in a pre-registration phase, in which the forecasted impact of a new medicine on clinical practice is estimated, has an important role in determining the extent of innovation and has allowed for assigning a partial score of 400 points to agomelatine. The remaining portion of the score (approximately 193 points) was obtained through the assessment of the clinical studies performed, which showed in their overall results that agomelatine may be a valid alternative therapy for major depression.

In assessing IAA-effectiveness, we took into consideration additional post-registration studies on efficacy, on tolerability and on endpoints. These are indeed secondary, but of primary importance for this kind of disorder, as they include quality of life, the effect on sleep architecture, the lack of damaging effects of a sexual nature, and the like. The representativeness of the sample presented was deemed adequate (approximately 580 patients treated with agomelatine). The same may be said for the types of studies, all RTC, both versus placebo, and versus the referenced drug. It is appropriate to stress that in two studies presented, the patients enrolled had a HAM-D $\geq 25$ basal value, therefore, a severe degree of the disorder. Moreover, in two studies agomelatine proved to be superior to the comparison drug either for the reduction 
of the HAM-D score or relative to the polysomnographic parameters as well as actigraphy findings and more generally for sleep architecture. The evaluation of no influence on sexual dysfunction during treatment with antidepressants was further confirmed by a comparison study versus paroxetine in 92 healthy volunteers with significantly superior results in the group treated with agomelatine. The findings reported above confirm good efficacy and tolerability of agomelatine compared to the placebo and to comparison medicines, both for the improvement of depression symptoms and for equally important aspects such as sleep architecture and disturbances of a sexual nature. However, it is appropriate to stress the absence of adequate data relative to the length of treatment (maximum 8 weeks, excluding one 6-month study) as well as data concerning subpopulations such as, for example, the elderly and/or patients with major depression also affected by other pathologies. If we look at the overall agomelatine assessment (IAA-efficacy + IAA-effectiveness), the score attributed to the maximum obtainable through entry into branch B for efficacy, appears to be improved overall. The improvement is due in part to confirmation of the medicine's efficacy and in part to the very low incidence and/or lack of serious adverse or potentially damaging side effects; this can be attributed to the peculiar action mechanism of agomelatine which is safer compared to the SSRI, particularly in some potentially serious conditions such as, for example, serotonergic syndrome.

\section{CONCLUSIONS}

This study reports an estimate of the degree of innovation for agomelatine (Valdoxan ${ }^{\circledR} /$ Thymanax $^{\circledR}$ ), performed through the Innovation Assessment Algorithm (IAA) on the basis of data supplied by Servier in support of its application for placement on the market, presented with a centralized procedure (IAAefficacy), and of additional post-registration studies (IAA-effectiveness). The Innovation Assessment Algorithm (IAA) allows one to attribute to each medicine an innovation value expressed in numerical terms, which derives from the sum of scores obtained by proceeding on the decision tree. Agomelatine obtained the score of 592.73 in the efficacy phase and 291.3 in the effectiveness phase; the total score for the two phases was 884, which corresponds to a good degree of innovation of the molecule.

\section{REFERENCE}

1. Caprino L, Russo P. Developing a paradigm of drug innovation: an evaluation algorithm. Drug Discovery Today 2006; 11: 999-1006; doi: 10.1016/j.drudis.2006.09.009

2. Millan MJ, Gobert A, Lejeune F, et al. The novel melatonin agonist agomelatine (S20098) is an antagonist at 5-hydroxytryptamine $2 \mathrm{c}$ receptors, blockade of which enhances the activity of frontocortical dopaminergic and adrenergic pathways. J Pharmacol Exp Ther 2003; 306: 954-64; doi: 10.1124/jpet.103.051797

3. Kasper S, Hamon M. Beyond the monoaminergic hypothesis: Agomelatine, a new antidepressant with an innovative mechanism of action. World J Biol Psychiatry 2009; 2: 1-11

4. Racagni G, Riva MA, Popoli M. The interaction between the internal clock and antidepressant efficacy. Int Clin Psychopharmacol 2009; 22: S9-S14; doi: 10.1097/01.yic.0000277957.75852.c7

5. Lôo H, Hale A, D'Haenen H. Determination of the dose of agomelatine, a melatoninergic agonist and selective 5-HT(2C) antagonist, in the treatment of major depressive disorder: a placebo-controlled dose range study. Int Clin Psychopharmacol 2002; 17: 239-47; doi: 10.1097/00004850-200209000-00004

6. Montgomery SA, Kasper S. Severe depression and antidepressants: focus on a pooled analysis of placebo-controlled studies on agomelatine. Int Clin Psychopharmacol 2007; 22: 283-91; doi: 10.1097/YIC.0b013e3280c56b13

7. Kennedy SH, Emsley R. Placebo-controlled trial of agomelatine in the treatment of major depressive disorder. Eur Neuropsychopharmacol 2006; 16: 93-100; doi: 10.1016/j.euroneuro.2005.09.002

8. Olié JP, Kasper S. Efficacy of agomelatine, a MT1/MT2 receptor agonist with 5-HT2C antagonistic properties, in major depressive disorder. Int J Neuropsychopharmacol 2007; 10: 661-73

9. European Medicine Agency. CHMP Assessment Report for Valdoxan. Doc.Ref.: EMEA/655251/2008.Available at: http://www.ema.europa.eu/

10. Goodwin GM, Rouillon F, Emsley R. Long term efficacy of agomelatine, a novel antidepressant, in the prevention of relapse in out-patients with major depressive disorder. Eur Neuropsychopharmacol 2007; 17(supp14): S361; doi: 10.1016/S0924-977X(07)70529-1 
11. Lemoine P, Guilleminault C, Alvarez E. Improvement in subjective sleep in major depressive disorder with a novel antidepressant, agomelatine: randomized, double-blind comparison with venlafaxine. J Clin Psychiatry 2007; 68: 1723-32; doi: 10.4088/JCP.v68n1112

12. Kennedy SH Rizvi S, Fulton K, Rasmussen J. A double-blind comparison of sexual functioning, antidepressant efficacy, and tolerability between agomelatine and venlafaxine XR. J Clin Psychopharmacol 2008; 28: 329-33; doi: 10.1097/JCP.0b013e318172b48c

13. Kasper S, Laigle L, Baylé F. Superior antidepressant efficacy of agomelatine versus sertraline: a randomised, double-blind study. Eur Neuropsychopharmacol 2008; 18(supp14): S336; doi: 10.1016/S0924-977X(08)70468-1

14. Sthal SM, Fava M, Trivedi MH, et al. Agomelatine in the treatment of major depressive disorder: an 8-week, multicenter, randomized, placebo-controlled trial. J Clin Psychiatry 2010; 71: 616-26; doi: 10.4088/JCP.09m05471blu

15. Hale A, Corral RM, Mencacci C, et al. Superior antidepressant efficacy results of agomelatine versus fluoxetine in severe MDD patients: a randomized, double-blind study. Int Clin Psychopharmacol 2010; 25: 305-14; doi: 10.1097/ YIC.0b013e32833a86aa

16. Kasper S, Hajak G, Wulff K, et al. Efficacy of the novel antidepressant agomelatine on the circadian rest-activity cycle and depressive and anxiety symptoms in patients with major depressive disorder: a randomized, double-blind comparison with sertraline. J Clin Psychiatry 2009; 71: 109-20; doi: 10.4088/JCP.09m05347blu

17. Quera-Salva MA, Hajak G, Philip P, et al. Comparison of agomelatine and escitalopram on nighttime sleep and daytime condition and efficacy in major depressive disorder patients. Int Clin Psychopharmacol 2011; 26: 252-62; doi: 10.1097/YIC.0b013e328349b117

18. Montejo AL. Better sexual acceptability of agomelatine (25 and $50 \mathrm{mg}$ ) compared with paroxetine (20 mg) in healthy male volunteers. An 8-week, placebo-controlled study using the PRSEXDQ-SALSEX scale. Int Clin Psychopharmacol 2010; 24: 111-20 